\title{
A method of fetal heart rate detection based on joint Time- Frequency analysis
}

\author{
Zhiwei Nie \\ School of Computer Science, Guangdong University of Technology \\ nzw2010@126.com
}

\begin{abstract}
Accurate fetal heart rate detection is the main task for fetal heart rate monitoring. To improve accuracy of fetal heart rate estimation, filtering processing would be taken for the collected fetal heart sound signals and then processed fetal heart sound signals would undergo time-frequency analysis with variational mode decomposition. Next, the variations obtained through decomposition would be used for kurtosis values calculation. Variations with smaller kurtosis values would be selected to calculate energy value of the signals and fetal heart rate could be estimated through limited peak-peak difference unit time. This method can not only estimate fetal heart rate very rapidly, but also reduce effects of noise on the estimated results of fetal heart rate when signal to noise ratio is quite low and the error is acceptable. Simulation experiment indicated that this method was of high accuracy in calculating fetal heart rate taking advantage of fetal heart sound signals under the circumstance of strong noise and was of great significance for the monitoring of fetal heart rate.
\end{abstract}

Key words: Fetal heart rate, variation mode decomposition, signal energy, kurtosis.

\section{INTRODUCTION}

Fetal heart rate refers to beating times of fetal heart per minute and its major content of fetal heart rate monitoring. It reflects vital signs of the fetus in uterus and is important indication to judge situation of fetus in uterus [1]. It's showed clinically that normal range of fetal heart rate is 120 to 160 time per minute and long-term exceeding 160 times or lower than 120 times per minute indicates fetal hypoxia for which reasons shall be figured out timely to prevent the fetal nerves from suffering irreversible damage [2].

At present, the most common procedures for fetal heart rate detection went as follow. Noise reduction through filtering shall be taken for the collected fetal heart sound signals and then heart beat times per minute shall be calculated based on position of peak value of signal's waveform after filtering [3]. During collection of fetal heart sound signals, noise such as the mother's heart sound would be unavoidably included and the signal noise ratio was low. Therefore, accurate calculation of fetal heart rate through conventional heart rate calculation was quite difficult [4].

Major steps for fetal heart rate detection algorithm included signal noise reduction such as short time fourier transform denoising [5], wavelet transform denoising [6], empirical mode decomposition denoising [7] and independent compo-nent analysis denoising [8] and fetal heart rate calculation. Denoising efficacy of these methods on nonlinear and unstable signals of fetal heart sounds was not very good [9] and when signal noise ratio was lower than certain threshold value, inaccurate calculation of fetal heart rate would be caused.

As a tool for time-frequency analysis, variational mode decomposition was of great application value on processing of audio signals, biological signals, mechanical fault analysis and other aspects [10]. Regarding how to extract signals required for fetal heart rate measurement from the complex fetal heart sound signals, this article calculated component kurtosis value to judge its relativity with fetal heart rate through optimization of variational mode decomposition and then selected proper variation as the input signals of fetal heart rate calculation, which greatly reduced amount of information and avoided unnecessary interference. It's of certain guidance on clinical monitoring of fetal heart rate. 


\section{PRINCIPLES OF FETAL HEART RATE DETECTION BASED ON VARIATION MODE DECOMPOSITION AND SHORT TIME ENERGY}

\section{Principle of variation mode decomposition}

Variation mode decomposition is a kind of time-frequency analysis of signals and widely applied in signal decomposing and denoising. The solving process is the solving of variational problems and its fundamental principle is to construct the following problems:

$$
\min _{\left\{u_{k}, w_{k}\right\}}\left\{\sum_{k}\left\|\partial_{t}\left[\left(\delta(t)+\frac{j}{\pi t}\right) * u_{k}(t)\right] e^{-j w_{k} t}\right\|_{2}^{2}\right\}
$$

And

$$
\mathrm{x}(t)=\sum_{k} u_{k}
$$

Among, value range of $\mathrm{k}$ is $\{1,2,3, \cdots, K\}$

This method can determine frequency centroid and bandwidth of each component through optimal solution of variation model with iteration during the process of obtaining decomposed variation, realizing adaptively frequency domain decomposition and effective decomposition of each component. The construction of objective function included three steps which came as follow.

(1) Analytic signal related to each mode would be calculated through Hilbert transform

$$
\left[\left(\delta(t)+\frac{j}{\pi t}\right) * u_{k}(t)\right]
$$

Among, $\mathrm{t}$ was time, $\delta(t)$ was impulse function, $u_{k}(t)$ was $\mathrm{k}$ component of signals.

(2) Exponential term was added to adjust each estimated center frequency and shift frequency spectrum of each modal to baseband

(3) Estimated application of bandwidth can get know of H Gaussian smoothing which was square of 2-norm of gradient

Among $\left\{u_{k}\right\}, k \in\{1,2,3, \cdots, K\}$ was the collection of mode function and $\left\{w_{k}\right\}, k \in\{1,2,3, \cdots, K\}$ was center frequency.

\section{Kurtosis}

Kurtosis was the fourth-order cumulant measuring features of stochastic signal and third order was inclination. General non-gaussian signals were measured by kurtosis and inclination, which reflected numerical statistics of distribution of vibration signals and normalized fourth central moment. Discretized formula can be indicated as:

$$
\mathrm{K}=\frac{1}{N} \sum_{i=1}^{N}\left(\frac{x_{i}-\bar{x}}{\sigma_{t}}\right)^{4}
$$

In the formula, $x_{i}$ was signal value and, $\bar{x}$ was mean signal value. $\mathrm{N}$ was sampling length and $\sigma_{t}$ was standard derivation. Probability density of high amplitude in the signal was enhanced and distribution deviation of signal amplitude was in the form of normal distribution. The normal curve was deflected or dispersed along with which kurtosis value increased. When there was beating points of heart sounds in the signal, the signal kurtosis was improved. This feature can be taken advantage of to judge signal components for proper gain of fetal heart rate. 


\section{STUDY OF FETAL HEART RATE DETECTION BASED ON JOINT TIME- FREQUENCY ANALYSIS}

Algorithm process was as shown in the figure and major procedure came as:

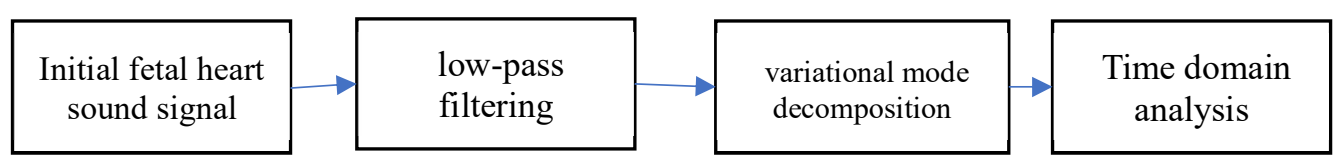

FIGURE 1. Flow chart of short time fetal heart rate detection

Step 1 Obtain fetal heart sound signals and carry out FFT transform of the original signal. Cut off signals whose frequency is lower than or equal to $250 \mathrm{~Hz}$ signal. For signals greater than $250 \mathrm{~Hz}$ and the setting was 0 , take FFT reverse transform to get signals after low-pass filtering with a cut-off frequency of $a=250 \mathrm{~Hz}$.

Step 2 Initialize data and set up component number of variational mode decomposition $\mathrm{K}=5$, penalty factor a $=2000$ and bandwidth $\mathrm{t}=0$

Step 3 Signals handled in line with step 1 would be decomposed in accordance to the parameters set in step 2 and calculate respectively kurtosis value of components

Step 4 In line with each kurtosis value, select several component signals with lower kurtosis value ri(t) as signals for fetal heart rate calculation

Step 5 Take signal energy formula for respective calculation of component ri(t) in step 4 for $\hat{r}(t)$

Step $6 \hat{r}(t)$ sequence should be calculated to make the curve of amplitude changed with time. Short time energy fetal heart rate could be estimated through number of qualified conditional points of the peak difference unit time.

\section{EXPERIMENTAL ANALYSIS}

To certify efficacy of fetal heart rate detection put forward in this article, fetal heart sound signal mimicked on MIT-BIH open database [11] shall be tested.

Various high-frequent information existed in the signals. Original signals shall be filtered to enable the signals passing low-pass filter with a cut-off frequency of $250 \mathrm{~Hz}$.

\section{Effect analysis of low-pass filter}

From the above, it can be figure that original signals shall be handled with filtering and short time Fourier transform shall be adopted for direct capture of signals not exceeding $250 \mathrm{~Hz}$. Then reverse transform would be taken for restoration.

Judging from the figure, the low frequency parts of signals after filtering were retained, reducing data of waveform. The spectrum diagram showed that there were several frequency signals for those under $100 \mathrm{~Hz}$ and after filtering, it's easier for time domain analysis.

\section{Variational mode decomposition and component selection}

Variational mode decomposition was taken for fetal heart sound signals after low-pass filtering. Then signals would be decomposed to certain components in line with parameters offered in step 2.

Variational mode decomposition can decompose the low frequency parts in the signal very well, which was the very part for the calculation of fetal heart rate. Variational mode decomposition can well avoid the defects in conventional time domain analysis such short time Fourier transform and wavelet transform can only capture given frequency signals or wavelet basis signals. For better selection of decomposed component for appropriate estimation of fetal heart rate, it's raised in this article that component kurtosis value can be compliance index for fetal heart rate estimation, kurtosis value calculation for each component.

The lower the kurtosis of signal component was, the stronger its periodicity was. Energy calculation indicated that the greater the energy was, the larger information was included. Finally, IMF1 was chosen to calculate component of fetal heart rate. 


\section{Calculation and comparison of fetal heart rate}

In line with maximal principles of energy and kurtosis ratio h(imf), IMF1 component would be chosen to be sequence for fetal heart rate calculation and data for each component value were as follow.

$$
\mathrm{h}(i m f)=\frac{E(i m f)}{K(i m f)}
$$

Among E(imf) was the energy value of component of signal imf and K(imf) was the kurtosis fo signal imf component.

TABLE 1. Energy and kurtosis ratio of each component

\begin{tabular}{cccccc}
\hline IMF & $\mathbf{1}$ & $\mathbf{2}$ & $\mathbf{3}$ & $\mathbf{4}$ & $\mathbf{5}$ \\
\hline $\mathrm{E} / \mathrm{K}$ & 43.0419 & 8.0164 & 10.9143 & 7.9154 & 36.1128 \\
\hline
\end{tabular}

Waveform of IMF1 component was indicated in the figure. It can be seen that besides several reference points, certain periodicity has emerged. It certified that maximal principle of the given energy and kurtosis ratio was effective. Accuracy (ACC), a diagnostic evaluation index for measurement testing or method was come up with according to literature [12].

$$
\mathrm{ACC}=\frac{T P}{T P+F P+F N}
$$

Among, TP (true positive) was the correctly detected number of fetal heart beats and FP (false positive) was the detected number of fetal heart beats which in fact was not. Meanwhile, FN (false negative) was the undetected but actual beated times.

ACC of low pass filtering and original input signal noise ratio (SNR) of $-15.1 \mathrm{~dB}$ in this article was compared respectively. The following figure was made.

For signals with different signal noise ratio, algorithm in this article was taken to measure heart rate and then formula was used to obtain ACC.

Make the following graph.

From the above graph can see that comparison of method used in this article and that offered in literature for fetal heart rate estimation and under the circumstance of high signal noise ratio of original fetal heart sound, accuracy rate of results differed slightly. When the signal suffered from much interference and the signal noise ratio was lower than $-15 \mathrm{~dB}$, method in this article can improve greatly than that in literature. When the signal noise ratio was $-25 \mathrm{~dB}$, accuracy rate of method in this article reached $90 \%$.

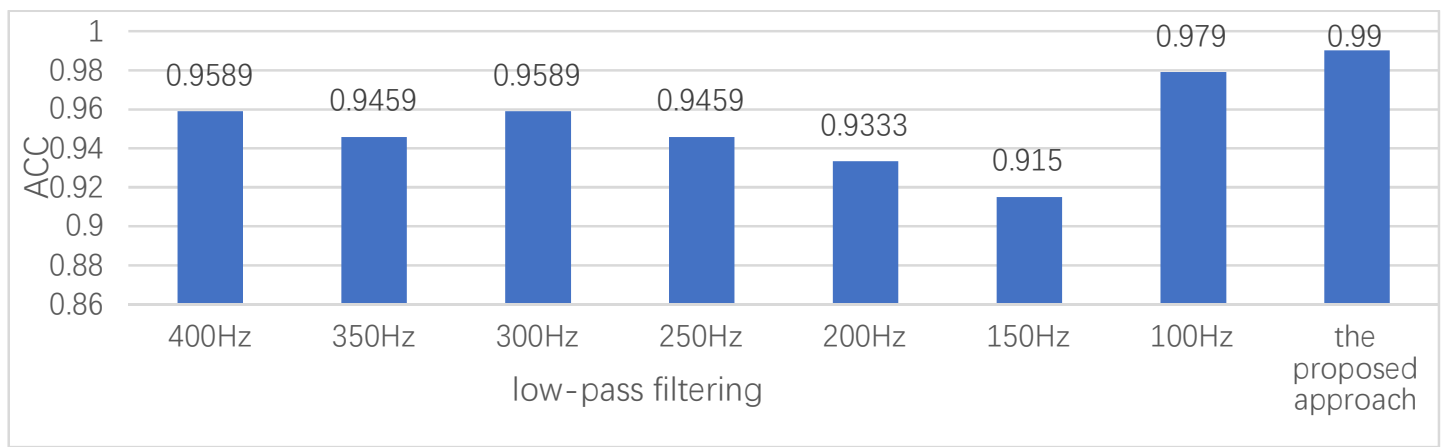

FIGURE 2. Comparison of low pass filtering wave of different cut-off frequency and method in this article 


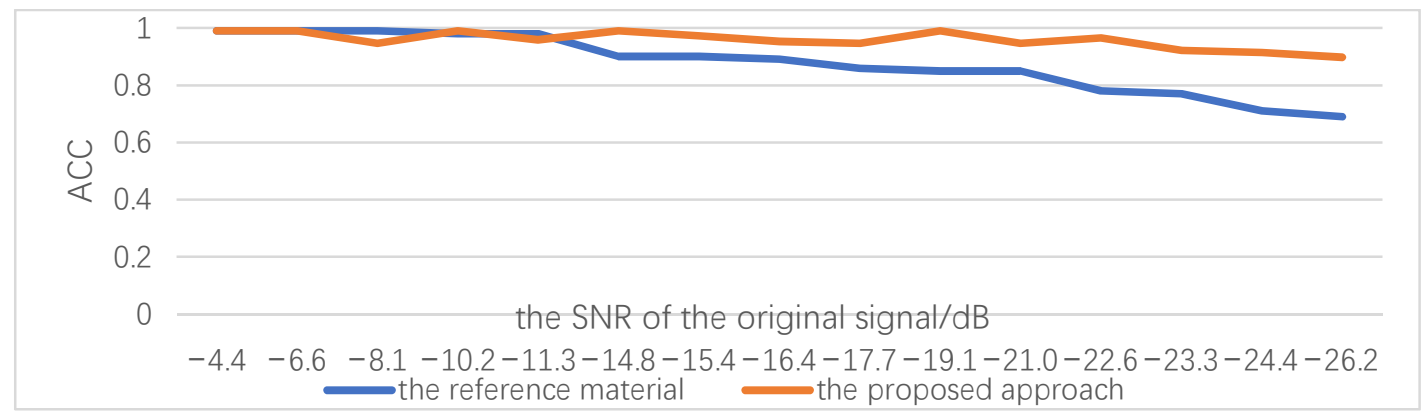

FIGURE 3. Comparison of methods in literature 12 and this article

\section{CONCLUSION}

When collecting fetal heart sound signals, there would be unavoidable noise disturbance which would lead to deviations in fetal heart rate calculation and incapability to accurately judge the fetus in the uterus. In consideration of this, fetal heart rate detection based on variational mode decomposition was presented and component selection was optimized. Control experiment was taken to verify the efficacy and practicability of the proposed method, laying good foundation for further monitoring of fetal heart.

\section{REFERENCES}

1. Liang Hu. System Design of Fetal Heart Rate Detection with Ultrasound Doppler [D]. Yunnan University, 2013.

2. Barqueropérez Ó, Santiagomozos R, Lillocastellano J M, et al. Fetal Heart Rate Analysis for Automatic Detection of Perinatal Hypoxia Using Normalized Compression Distance and Machine Learning: [J]. Frontiers in Physiology, 2017, 8.

3. Ming Dai, Kai Zhan, Xin Chen, etc. Design and Realization of a Portable Doppler Fetal Heart Detection System [J]. Journal of Biomedical Engineering Research, 2017, 36(2): 137-142.

4. Papadimitriou S, Gatzounas D, Papadopoulos V, et al. Fetal heart rate signal denoising by processing the wavelet transform modulus maxima[C]// Engineering in Medicine and Biology Society, 1996. Bridging Disciplines for Biomedicine. Proceedings of the, International Conference of the IEEE. IEEE, 1996:1029-1030 vol.3.

5. Xiao H, Chen X, Luo K, et al. A Study on the Measurement Arithmetic for Ultrasonic Doppler Fetal Heart Rate Based on FFT[J].

6. Karvounis E C, Papaloukas C, Fotiadis D I, et al. Fetal heart rate extraction from composite maternal ECG using complex continuous wavelet transform[C]// Computers in Cardiology. IEEE, 2004:737-740.

7. T,arălungă D, Ungureanu M, Hurezeanu B, et al. Fetal Heart Rate Estimation from Phonocardiograms Using an EMD Based Method[C]// RECENT ADVANCES in COMPUTER SCIENCE. 2015.

8. Zulkifli S. Investigation of Independent Component Analysis (ICA) To Fetal Heart Rate Using Photoplethysmography (PPG)[J]. 2008.

9. Bewley S, Braillon A. Electronic fetal heart rate monitoring: we need new research approaches. [J]. BMJ, 2018, 360: k658.

10. Dragomiretskiy K, Zosso D. Variational Mode Decomposition[J]. IEEE Transactions on Signal Processing, 2014 62(3):531-544.

11. Goldberger AL, Amaral LAN, Glass L, Hausdorff JM, Ivanov PCh, Mark RG, Mietus JE, Moody GB, Peng CK, Stanley HE. PhysioBank, PhysioToolkit, and PhysioNet: Components of a New Research Resource for Complex Physiologic Signals. Circulation 101(23): e215-e220

12. De L L, De M B, Vandewalle J. Fetal electrocardiogram extraction by blind source subspace separation[J]. IEEE Transactions on Biomedical Engineering, 2002, 47(5):567-572. 